\title{
The effects of new dentures on facial aesthetics
}

\author{
The effect of increasing vertical dimension of occlusion on facial aesthetics

\section{N. K. Mohindra and J. S. Bulman Br Dent J 2002; 192: 164-168}

\section{Aim}

To investigate the effect of increasing the vertical dimension of occlusion on facial aesthetics.

\section{Setting}

General practice.

\section{Method}

Questionnaires were sent to 96 patients who had been treated in the practice during the period of July 1998 to December 2000, resulting in an overall 72\% response rate. All these patients had had their occlusal vertical dimension increased. Photographs of patients were taken before, during and after treatment. The questionnaire asked their opinion on the effects of the treatment on their facial features. To obtain an objective view to substantiate the opinions of the patients, a panel of five judges reviewed the before and after photographs and filled in their own questionnaires.

\section{Results}

Of the patients who responded to the questionnaire, $79.7 \%$ said they looked younger after the treatment. The panel thought 81.2\% of the patients treated whose photographs they reviewed looked younger.

\section{Conclusion}

Increasing the vertical dimension of occlusion can have far reaching effects on facial aesthetics, not just on the peri-oral areas but on the whole face.

\section{IN BRIEF}

- This study looked at 96 patients whose facial aesthetics had been improved by increasing the vertical dimension of occlusion.

- To substantiate the subjective view of the patients, an independent panel also looked at the patients' pictures.

- According to the views of the patients and the panel, nearly $80 \%$ of patients were thought to look 5 to 20 years younger. The improvement related to the whole range of facial features (eyes, nose, cheeks, lips and skin) not just the lower face.

- This treatment is non-surgical, and gives the natural improvement which some patients prefer to cosmetic surgery.

- This improvement in facial aesthetics is a truly anti-ageing process and opens new avenues for further research.

\section{COMMENT}

Dr. Mohindra has a long standing interest in constructing complete dentures. In particular he has focussed on how best to determine the vertical dimension of occlusion. This work resulted in a paper, published in the British Dental Journal in 1996, entitled 'A preliminary report on the determination of the vertical dimension of occlusion using the principle of the mandibular position in swallowing: This second paper is a logical development of his initial work and looks at the effect of improving the vertical dimension of occlusion on facial appearance.

A questionnaire was used to ask patients whether their new dentures had made them look younger and if so, by how many years. In addition they were asked whether any of their facial features had improved (lips: jaw line: eyes: chin: skin). A panel of five judges were asked to make similar assessments using before and after photographs.

The majority of people who answered the questionnaire said they looked younger after treatment. A similar view was expressed by the panel. Perhaps not surprisingly, the response to the question as to how many years younger they looked was more modest from the patients but more generous from the panel. The suggestion that new complete dentures will make a person look younger could raise unrealistic expectations. However some of the patients stated that they did not particularly want to look younger, but rather wanted to look good for their age. This, whilst still fraught with pitfalls, could provide a more realistic goal.

An interesting finding is that the patients felt that new dentures had produced improvements in various facial features, and that these were not just confined to the immediate peri-oral tissues, such as the lips. Twenty-eight percent thought that their eyes had improved whilst a similar percentage thought their skin had improved. The panel's findings were similar. Such results indicate that the provision of new dentures can have a much wider impact than is traditionally taught.

Dr. Mohindra is to be congratulated on continuing to investigate his work. I look forward to his next paper. For example, in constructing the replacement dentures more than the vertical dimension of occlusion will have been changed. It would be interesting to know the extent to which this change alone produced improvements in appearance and the contribution made by other factors, such as increased lip support.

David Davis, Senior Lecturer, Honorary Consltant, Department of Prosthetic Dentistry, GKT Dental Institute 